\title{
AUTOSCREEN - a novel platform concept for automated high throughput high end microscopy
}

Klaus Palme ${ }^{1}$, Rainer Uhl

${ }^{1}$ Institute of Biology II/Molecular Plant Physiology, Faculty of Biology, Albert-LudwigsUniversity of Freiburg, Schänzlestrasse 1, D-79104 Freiburg, Germany; Centre for Biological Systems Analysis, Albert-Ludwigs-University of Freiburg; Habsburgerstr. 49, D-79104 Freiburg, Germany; Freiburg Institute of Advanced Sciences (FRIAS), Albert-LudwigsUniversity of Freiburg, Albertstrasse 19, D-79104 Freiburg, Germany; Centre for Biological Signalling Studies (BIOSS), Albert-Ludwigs-University of Freiburg, Albertstrasse 19, D-79104 Freiburg, Germany; Freiburg Initiative for Systems Biology (FRISYS), Albert-LudwigsUniversity of Freiburg, Schänzlestrasse 1, 79104 Freiburg, Germany;

²Biolmaging Zentrum der Ludwig Maximilians Universität München, Großhaderner Straße 24 , 82151 Martinsried; TILL I.D. GmbH, Bahnhofstraße 89, 82166 Gräfelfing, Germany.

The overall objective of the AUTOSCREEN project was the establishment of an innovative, automated screening instrument for high-throughput and high-content screens. AUTOSCREEN was planned to allow standardized, robust, automated, ultrasensitive, highresolution analysis of cellular components at cellular and subcellular resolution. The generic technology was thought to be widely applicable in biomedical research and commercially relevant. In our view such instrumentation is urgently needed by the genome research community. The availability of complete genome sequences for biomedical research and the difficulty to obtain functional knowledge on the huge number of genes calls for better, robust and automated gene function screening technology at highest resolution. AUTOSCREEN is timely and presents important opportunities for high-throughput and high-content assays at a genome-wide scale. The AUTOSCREEN SMEs have established such a widely applicable robot-automated screening tool for high-throughput and high-content cell-based assays and demonstrate its suitability for high-resolution in situ techniques. We expect that AUTOSCREEN will arm academia and industry with new, innovative technologies, having the potential to revolutionize high-content screening. 